\title{
INTEGRAL EQUATIONS WITH DIFFERENCE KERNELS ON FINITE INTERVALS $\left({ }^{1}\right)$
}

\author{
BY \\ A. LEONARD AND T. W. MULLIKIN $\left(^{2}\right)$
}

1. Introduction. Considerable significance is attached to integral equations on the real line whose kernels depend upon the difference of the arguments. Such problems for the infinite line are often readily solved by use of the Fourier transform.

For the half-line, the use of the Fourier transform requires some sophisticated function theory. Methods were developed by Wiener and Hopf [10] for which there is a vast literature devoted to its development and application. A very comprehensive study and bibliography are given in a paper by Krein [4].

In this paper we wish to present a technique for the study of such integral equations on a finite interval. Rather than attempt a development for kernel functions of various general classes, we consider those of a particular form which arise in the theories of neutron transport [2], radiative transfer [1], gas dynamics [11], [12], and electromagnetic wave refraction [3]. Very complete results are obtained in the form of simple quadratures and rapidly convergent Fredholm equations. We leave to a future paper the investigation of general classes of kernel functions [14]- [16].

Most of the details of this paper were worked out in a previous paper [6] devoted to the study of Chandrasekhar's $X$ and $Y$ equations which arise in the theory of radiative transfer [1]. We wish here to show that the methods exploited there can be used as well to obtain a spectral analysis of certain Fredholm operators. Our methods make use of the theory of singular integral equations and analytic continuation. The Laplace transform is explicitly used to represent the kernel function, but it plays a minor role in the development.

\footnotetext{
Received by the editors December 15, 1963.

(1) This research is sponsored by the United States Air Force under Project RANDContract No. AF 49(638)-700 monitored by the Directorate of Development Planning, Deputy Chief of Staff, Research and Development, Hq USAF. Views or conclusions contained in this Memorandum should not be interpreted as representing the official opinion or policy of the United States Air Force.

$\left({ }^{2}\right)$ Present address: Division of Mathematical Sciences, Purdue University, Lafayette, Indiana.
} 
We wish to draw special attention to two points. The first is the method of derivation of equation (3.5) below. A similar equation can be derived for any kernel function with a Laplace transform representation. The second point is the consistent use of analytic continuation which is prominent in the detailed calculations of our previous paper [6].

2. Fredholm equations. We shall study integral equations of the following type

$$
f(x)=c \int_{0}^{r} k(|x-y|) f(y) d y+g(x)
$$

for $0 \leqq \tau<\infty$ and $0 \leqq c<\infty$. The kernel functions will be of the type

$$
k(x)=\int_{\alpha}^{\infty} \psi(t) \exp [-x t] \frac{d t}{t} \quad(0 \leqq \alpha<\infty)
$$

where the function $\psi$ is nonnegative $\left({ }^{3}\right)$ on $[\alpha, \infty)$, satisfies a Hölder condition on $(\alpha, \infty)$ and satisfies the condition

$$
\int_{\alpha}^{\infty} \psi(t) \frac{d t}{t^{2}}=\frac{1}{2}
$$

In the theories of neutron transport and radiative transfer, $\psi$ is usually a polynomial in $1 / t$ and $\alpha=1$. With certain assumptions, $\psi$ is $\exp \left[-1 / t^{2}\right]$ and $\alpha=0$ in Couette flow problems in gas dynamics. If we take $\psi$ $=t / \sqrt{ }\left(t^{2}-1\right)$ and $\alpha=1$ we have the MacDonald kernel which arises in electromagnetic wave refraction.

We write (2.1) in abbreviated notation as

$$
f=c \Lambda_{r}(f)+g .
$$

It is easy to show [7] that $\Lambda_{r}$ is a compact, positive definite, self-adjoint operator in $L_{2}[0, \tau]$. By a simple extension of a result observed in a previous paper [7], we obtain good upper bounds for the maximum eigenvalue $\mu_{1}(\tau)$ of $\Lambda_{r}$ as

$$
\begin{aligned}
& \mu_{1}(\tau) \leqq 2 \int_{\alpha}^{\infty} \frac{\psi(s)}{s^{2}+\beta^{2}} d s, \\
& \beta \tan \frac{\beta \tau}{2}=s, \quad 0<\frac{\beta \tau}{2} \leqq \frac{\pi}{2} .
\end{aligned}
$$

We have two objectives in this paper. The first objective is to present a procedure for the precise computation of eigenvalues and eigenfunctions of $\Lambda_{r}$, that is, solutions to

( $\left.{ }^{3}\right)$ The restriction to positivity is made to simplify the following discussion. The extension of the results to arbitrary $\psi$ satisfying a Hölder condition is relatively straightforward (see [5]). 


$$
\mu_{i} \phi_{i}=\Lambda_{r}\left(\phi_{i}\right) \quad(i=1,2, \cdots) .
$$

We do this by first solving (2.4) for special functions $g$.

When $c \mu_{1}<1$, equation (2.4) can be solved for $f$ by iteration. The nearer $c \mu_{1}$ is to 1 , the slower the convergence. Our second objective is the presentation of a procedure for solving (2.4) which is less sensitive to conditions on $c$. To do this we first restrict the function $g$ in (2.4) to exponential functions. Solutions for more general $g$ are obtained by use of the Laplace transform [13].

We shall need the following obvious result.

Theorem 1. For $c \mu_{i} \neq 1(i=1,2, \cdots)$, the solution $J$ to the equation

$$
J(x, z)=c \Lambda_{\tau}(J)(x, z)+\exp [-x z]
$$

is unique and an analytic function of $z$ for $|z|<\infty$.

3. Linear singular equations. To make full use of the analytic dependence of $J$ in (2.7) upon the parameter $z$, we proceed to show that $\Lambda_{r}(J)$ can be expressed in terms of integrals on the parameter $z$ in $J$. This result and analyticity of $J$ in the variable $z$ leads to singular integral equations and linear constraints.

We apply $\Lambda_{r}$ to (2.7) to obtain the equation for $\Lambda_{r}(J)$

$$
\begin{aligned}
& \Lambda_{\tau}(J)(x, z)-c \Lambda_{\tau}\left(\Lambda_{\tau}(J)\right)(x, z) \\
& =\int_{\alpha}^{\infty}\left[\frac{\exp [-x z]-\exp [-x t]}{t-z}\right. \\
& \left.\quad+\frac{\exp [-x z]-\exp [-\tau(z+t)+x t]}{t+z}\right] \frac{\psi(t) d t}{t} .
\end{aligned}
$$

From (2.7) we readily obtain the fact that

$$
\begin{aligned}
& \Lambda_{r}(J)(x, z) \\
& \quad=\int_{\alpha}^{\infty}\left[\frac{J(x, z)-J(x, t)}{t-z}\right. \\
& \left.\quad+\frac{J(x, z)-J(x,-t) \exp [-\tau(z+t)]}{t+z}\right] \frac{\psi(t)}{t} d t .
\end{aligned}
$$

Also from (2.7) it follows readily that

$$
J(\tau-x, t)=\exp [-\tau t] J(x,-t) .
$$

Combining (2.7), (3.2) and (3.3), we obtain the following result.

Theorem 2. For $c \mu_{i}(\tau) \neq 1$, the unique solution to (2.7) also satisfies the equation 


$$
\begin{aligned}
J(x, z)=\exp [-x z]+c \int_{\alpha}^{\infty} & {\left[\frac{J(x, z)-J(x, t)}{t-z}\right.} \\
& \left.+\frac{J(x, z)-\exp [-\tau z] J(\tau-x, t)}{t+z}\right] \frac{\psi(t)}{t} d t .
\end{aligned}
$$

The variable $z$ is any complex number in $|z|<\infty$ and $x$ is a parameter restricted by $0 \leqq x \leqq \tau$.

We now restrict the complex variable $z$ by $1 / z \notin[-1 / \alpha, 1 / \alpha]$. Then we can rewrite (3.4) as

$$
\begin{aligned}
\lambda(z) J(x, z)= & \exp [-x z]-c \int_{\alpha}^{\infty} \frac{J(x, t) \psi(t)}{t-z} \frac{d t}{t} \\
& -\exp [-\tau z] c \int_{\alpha}^{\infty} \frac{J(\tau-x, t) \psi(t)}{t+z} \frac{d t}{t}
\end{aligned}
$$

where

$$
\lambda(z, C) \equiv 1-2 c \int_{\alpha}^{\infty} \frac{\psi(t)}{t^{2}-z^{2}} d t .
$$

If we specialize $\psi$ and $\alpha$ and evaluate (3.5) at $x=0$ and at $x=\tau$ and denote $J(0, z)$ by $X(z)$ and $J(\tau, z)$ by $Y(z)$, we obtain equations for Chandrasekhar's $X$ and $Y$ functions. These equations were studied in a previous paper [6]. All of the analysis given there applies to (3.5) when proper account is taken of the fact that $x$ is no longer restricted to 0 and $\tau$. We shall use the results of this previous analysis without reproducing any of the computations.

We first define functions $F$ and $G$ by

$$
F(x, z) \equiv J(x, z)+J(\tau-x, z), G(x, z) \equiv J(x, z)-J(\tau-x, z) .
$$

Then (3.5) leads to uncoupled equations for $F$ and $G$. These equations give rise to linear constraints since analyticity of $J$ requires the vanishing of the right-hand side of (3.5) at all zeros of $\lambda$ for $1 / z \notin[-1 / \alpha, 1 / \alpha]$. In addition, we obtain linear singular equations for $F$ and $G$ by adding the limits in (3.5) as $z$ approaches the cut from above and below, as given by Plemelj's formulae $[8$, p. 42$]$. We, then, invert the singular operator by standard methods [8, Chapter 14] and, finally, by analytic continuation, we achieve the desired solution.

To present the solution to (3.5) we need a classification of the roots of the function $\lambda$ for $1 / z \notin[-1 / \alpha, 1 / \alpha]$. We have the following possibilities which depend upon values of $c\left({ }^{4}\right)$.

$\left({ }^{4}\right)$ If we allow $\psi$ to take on negative values, other possibilities arise, e.g., $\lambda(z)$ may have two pair of zeros $\pm z_{0}, \pm z_{1}$. 
(i) If $0 \leqq 2 c \int_{\alpha}^{\infty} \psi(t) /\left(t^{2}-\alpha^{2}\right) d t \leqq 1$, then

$$
\lambda(z) \neq 0 \text { for } 1 / z \notin[-1 / \alpha, 1 / \alpha] \text {. }
$$

(3.8) (ii) If $1 / 2 \int_{\alpha}^{\infty} \psi(t) /\left(t^{2}-\alpha^{2}\right) d t<c \leqq 1$, then

$$
\lambda\left( \pm z_{0}\right)=0 \text { for some } z_{0}, 0 \leqq z_{0}<\alpha .
$$

(iii) If $1<c<\infty$, then

$$
\lambda\left( \pm z_{0}\right)=0 \text { for some } z_{0}=i \nu_{0}, 0 \leqq \nu_{0}<\infty .
$$

Since the first possibility (i) seems to occur physically only in very special cases and since there exist no eigenvalues for this case, we will offer no further discussion on this possibility other than to mention that the following results for case (ii) may be easily modified to give the solution corresponding to case (i) (see [6]).

Now by a slight modification and rearrangement of results of our previous paper [6] we find the solution of (2.7) for case (ii) of (3.8). In terms of functions to be defined below, we have $F$ given by

$$
\begin{aligned}
F(x, z)=H(z)\left[P(x, z)-c \int_{\alpha}^{\infty} \frac{\psi(t)[P(x, t)-P(x, z)]}{H(t) M(t)[t-z]} \frac{d t}{t}\right. \\
\left.+\frac{z_{0}^{2}}{v(1-c) N\left(z_{0}\right)} \frac{P(x, z)-P\left(x, z_{0}\right)}{z-z_{0}}\right] .
\end{aligned}
$$

By replacing $P$ by $Q$ we obtain the result for the function $G$.

The various functions in (3.9) are defined as follows. The function $H$ is given by

$$
\begin{aligned}
& H(z)=\frac{z+\alpha}{z+z_{0}} \exp \left[\int_{\alpha}^{\infty} \frac{\theta(t)}{z+t} d t\right], \quad z \notin(-\infty,-\alpha) \text { and } z \neq-z_{0}, \\
& \theta(t)=\frac{1}{\pi} \tan ^{-1} \frac{c \pi \psi(t)}{t \lambda_{0}(t)} \quad(\theta(\alpha)=1 \text { and } \theta(\infty)=0), \\
& \lambda_{0}(t)=1-2 c \int_{\alpha}^{\infty} \frac{\psi(s)-\psi(t)}{s^{2}-t^{2}} d s-\frac{c \psi(t)}{t} \ln \frac{t+\alpha}{t-\alpha} \quad(\alpha \leqq t<\infty) .
\end{aligned}
$$

The functions $M$ and $N$ are given by

$$
M(t)=\left[\lambda_{0}(t)\right]^{2}+\left[\frac{\pi c \psi(t)}{t}\right]^{2} \quad(\alpha \leqq t<\infty),
$$


and

$$
N(z)=-z \exp \left[z \int_{\alpha}^{\infty} \frac{(\theta(t)-1)}{(z-t)} \frac{d t}{t}\right], \quad z \notin[\alpha, \infty),
$$

with

$$
(1-c)\left(z^{2}-z_{0}^{2}\right) N(z) N(-z)=\left(z z_{0}\right)^{2} \lambda(z)
$$

The functions $P$ and $Q$ are given by

$$
\begin{aligned}
& P=h_{1}+C h_{2}, \\
& Q=h_{3}+D h_{4},
\end{aligned}
$$

where the $h$ functions satisfy the Fredholm equations

$$
\begin{aligned}
& h_{1}=-L\left(h_{1}\right)+\exp [-x z]+\exp [(x-\tau) z], \\
& h_{2}=-L\left(h_{2}\right)+\frac{N(-z)}{z} \exp [-\tau z], \\
& h_{3}=L\left(h_{3}\right)+\exp [-x z]-\exp [(x-\tau) z], \\
& h_{4}=L\left(h_{4}\right)+\frac{N(-z)}{z} \exp [-\tau z] .
\end{aligned}
$$

The operator $L$ in (3.11) is defined by

$$
L(h)=\exp [-\tau z] \frac{v(1-c) N(-z)}{z_{0}} \int_{\alpha}^{\infty} \frac{\psi(t) h(t)\left(t-z_{0}\right)}{H(t) M(t)(t+z)} \frac{d t}{t^{2}} .
$$

We show in [6] that the equations (3.11) can be solved by iteration to converge uniformly, since in this norm

$$
\|L\| \leqq \exp [-\alpha \tau]\left(1-\frac{\sqrt{ }(1-c)}{z_{0} \int_{\alpha}^{\infty}(1-\theta(t)) \frac{d t}{t^{2}}}\right)<1, \quad(\alpha>0) .
$$

This result holds for all $c$ and $\tau(0 \leqq c<\infty, \tau<\infty)$. For $c>1$ the root $z_{0}$ is pure imaginary but it follows readily from (3.10) that $\left(t-z_{0}\right) / H(t)$ is real-valued. Whether $L$ is contracting when $\alpha=0$ depends on $\psi$ (cf. [12]).

The above quadrature formula $(3.10)$ for the $H$ function gives a solution to Chandrasekhar's $H$ equation [1, Chapter 5] which is analytic in $\operatorname{Re}(z)>0$. This function is the Laplace transform of a certain solution to equation (2.1) with $g=0$ for the half-line, $\tau=\infty,[4$, p. 273].

The constants $C$ and $D$ are determined by the requirement that $F$ and $G$ be analytic at the zeros of $\lambda$. They are functions of the parameter $x$ and for $z_{0} \neq 0$ are given by 


$$
\begin{aligned}
C & =\frac{\exp \left[-z_{0} x\right]+\exp \left[z_{0}(x-\tau)\right]-L\left(h_{1}\right)\left(z_{0}\right)-\exp \left[-z_{0} \tau\right] L\left(h_{1}\right)\left(-z_{0}\right)}{\frac{N\left(z_{0}\right)-\exp \left[-z_{0} \tau\right] N\left(-z_{0}\right)}{z_{0}}+L\left(h_{2}\right)\left(z_{0}\right)+\exp \left[-z_{0} \tau\right] L\left(h_{2}\right)\left(-z_{0}\right)} \\
& \equiv \frac{u(x, c, \tau)}{m(c, \tau)}, \\
D & =\frac{\exp \left[-z_{0} x\right]-\exp \left[z_{0}(x-\tau)\right]+L\left(h_{3}\right)\left(z_{0}\right)-\exp \left[-z_{0} \tau\right] L\left(h_{3}\right)\left(-z_{0}\right)}{\frac{-N\left(z_{0}\right)-\exp \left[-z_{0} \tau\right] N\left(-z_{0}\right)}{z_{0}}-L\left(h_{4}\right)\left(z_{0}\right)+\exp \left[-z_{0} \tau\right] L\left(h_{4}\right)\left(-z_{0}\right)} \\
& \equiv \frac{v(x, c, \tau)}{n(c, \tau)} .
\end{aligned}
$$

We merely remark, at this time, that the above constants for the limiting case, $z_{0}=0$, may be easily calculated from (3.12).

Two comments about computations are in order. First, it will be shown in a subsequent paper that the resolvent kernels for the operators $(I \pm L)$ have simple expressions in terms of $h_{2}$ and $h_{4}$, thereby giving an easy computation of $h_{1}$ and $h_{3}$ for different values of the parameter $x$. Second, in many applications integrals over the interval $[\alpha, \infty)$ can be transformed to integrals over a finite interval [6], [12].

4. Spectral analysis. For case (iii), the previous results, (3.9) to (3.12), hold with $z_{0}$ pure imaginary but with the following exception. For certain values of $c$ ( $\tau$ fixed), $m$ (or $n$ ) is zero and the solution to (2.7) is unbounded for all finite $x$ and $z$. Let these values of $c$ be denoted by $c_{i}(i=1,2, \ldots)$. Then, by Theorem 1 , the homogeneous equation (2.6) has a solution for each $c=c_{i}$ with

$$
\mu_{i}=\frac{1}{c_{i}}
$$

Furthermore, it is easily seen that $u$ (or $v$ ) is proportional to the corresponding eigenfunction, $\phi_{i}$, and that the even eigenfunctions belong to the singularities of $C$ and the odd eigenfunctions belong to the singularities of $D$. Therefore, we have, in essence, achieved a spectral analysis of $\Lambda_{r}$. We summarize this result by the following theorem.

Theorem 3. For a fixed $\tau$, let $\left\{c_{i}\right\}_{i=1}^{\infty}$ be the set of values of $c$ for which either

$$
m(c, \tau)=0
$$

or

$$
n(c, \tau)=0
$$


Then, for each $c_{i}$ the homogeneous equation (2.6) has a solution with

$$
\mu_{i}=\frac{1}{c_{i}}
$$

If (4.2) is satisfied, the corresponding eigenfunction, $\phi_{i}$, is given by

$$
\phi_{i}=\cos \left[\left|z_{0}\right|(\tau / 2-x)\right]-\operatorname{Re}\left\{\exp \left[z_{0} \tau / 2\right] L\left(h_{1}\right)\left(z_{0}\right)\right\}
$$

or, if (4.3) is satisfied, $\phi_{i}$ is given by

$$
\phi_{i}=\sin \left[\left|z_{0}\right|(\tau / 2-x)\right]+\operatorname{Im}\left\{\exp \left[z_{0} \tau / 2\right] L\left(h_{3}\right)\left(z_{0}\right)\right\} .
$$

The normalization is arbitrary. By (3.8), $z_{0}$ depends on $c_{i}$.

The product $m(c, \tau) n(c, \tau)$ is clearly the counterpart of the Fredholm determinant $D(c)$ which arises in the classical theory of Fredholm kernels [9]. Although the dependence of $m(c, \tau) n(c, \tau)$ on $c$ is very complicated, it is essentially given in closed form, whereas, in the general case of a Fredholm kernel, the computation of $D(c)$ can only be carried out in the form of a MacLaurin's series in which the higher order terms become progressively more cumbersome.

5. Other difference kernels. Although we have presently limited our discussion to kernels of the type given by (2.2), we hope, in the future, to treat other classes of kernel functions with the methods of this paper or variations thereof. For the cases considered in this paper, the contours in the $z$-plane reduced to integrals on the real line $[\alpha, \infty]$ (see, for example, (3.5)). In other cases, contours off the real line will probably be encountered.

\section{References}

1. S. Chandrasekhar, Radiative transfer, Dover, New York, 1960.

2. B. Davison, Neutron transport theory, Oxford Univ. Press, London, 1957.

3. G. A. Grinberg and V. A. Fok, On the theory of boundary refraction of electromagnetic waves, investigations on the propagation of radio waves, Izdat. Akad. Nauk SSR, Sb. 2, Moscow, $1948 ;$ p. 69.

4. M. G. Krein, Integral equations on a half-line with kernel depending upon the difference of the arguments, Amer. Math. Soc. Transl. 22 (1962), 163-288.

5. A. Leonard and T. W. Mullikin, A spectral analysis of the anisotropic neutron transport kernel in slab geometry with applications, J. Math. and Phys. 5 (1964), 399-411.

6. T. W. Mullikin, “Chandrasekhar's $X$ and $Y$ functions, Trans. Amer. Math. Soc. 113 (1964), 316-332.

7. Estimates of critical dimensions of spherical and slab reactors, J. Math. Anal. Appl. 5 (1962), 184.

8. N. I. Muskhelishvili, Singular integral equations, Noordhoff, Groningen, 1953.

9. F. G. Tricomi, Integral equations, Interscience, New York, 1957.

10. N. Wiener and E. Hopf, Über eine Klasse Singularer Integralgleichungen, S.-B. Preuss. Akad. Wiss. (1931), 696.

11. D. R. Willis, Comparison of kinetic theory analyses of linearized Couette flow, Phys. Fluids 5 (1962), 127. 
$12\left(^{5}\right)$ A. Leonard and T. Mullikin, An application of singular integral theory to a linearized problem in Couette flow, Ann. Phys. 30 (1964), 235-248.

13. _ Green's functions for one-velocity neutron transport in a one-dimensional slab and sphere, Proc. Nat. Acad. Sci. U.S.A. 52 (1964), 683-688.

14. G. F. Carrier, 50 Jahre Grenzschichtforschung (Edited by N. Gortler and W. Tollmein), Vieweg: Braunschweig, 1955, 13-20.

15. R. Latter, Approximate solutions for a class of integral equations, Quart. Appl. Math. 16 (1958), 21-31.

16. V. Huston, Asymptotic solutions of integral equations with convolution kernels, Proc. Edinburgh Math. Soc. 14 (1964/1965), 5-19.

\section{The RAND Corporation,}

Santa Monica, California

(5) Reference 12 and following references added in proof. 